

\title{
Les collections naturalistes: une ressource essentielle pour la science du XXIe siècle
}

Roseli Pellens

\section{To cite this version:}

Roseli Pellens. Les collections naturalistes: une ressource essentielle pour la science du XXIe siècle. Les collections naturalistes dans la science du XXIe siècle: une ressource durable pour la science ouverte 2021, 2021. hal-03409131

\section{HAL Id: hal-03409131 \\ https://hal.science/hal-03409131}

Submitted on 29 Oct 2021

HAL is a multi-disciplinary open access archive for the deposit and dissemination of scientific research documents, whether they are published or not. The documents may come from teaching and research institutions in France or abroad, or from public or private research centers.
L'archive ouverte pluridisciplinaire HAL, est destinée au dépôt et à la diffusion de documents scientifiques de niveau recherche, publiés ou non, émanant des établissements d'enseignement et de recherche français ou étrangers, des laboratoires publics ou privés. 


\title{
Chapitre 1
}

\section{Les collections naturalistes dans la science du $\mathrm{XXI}^{\mathrm{e}}$ siècle}

\author{
Roseli Pellens \\ Institut de Systématique, Évolution, et Biodiversité, Muséum national d'Histoire naturelle, \\ CNRS, Sorbonne Université, EPHE, UA, Paris, France
}

En ce début du $\mathrm{XXI}^{\mathrm{e}}$ siècle, nous traversons une crise environnementale majeure (Hoekstra et al. 2012 ; Hoekstra et Wiedmann 2014 ; Steffen et al. 2015). Le dérèglement climatique, la perte colossale de diversité biologique (Barnosky et al. 2011, 2012 ; Ceballos et al. 2015) et culturelle (Amano et al. 2014) ainsi que l'émergence et la dissémination de nouvelles maladies (Carlson 2020) sont quelques-unes des facettes les plus visibles de cette crise. Du fait de son ampleur globale, elle génère une nouvelle dynamique dans les négociations internationales (voir la Convention de la diversité biologique, Convention-cadre des Nations unies sur les changements climatiques), sans pour autant permettre à ce jour une évolution significative vers un modèle de production et de consommation à même de limiter ses effets.

Cette situation crée une demande importante vers la communauté scientifique, qui est appelée à produire des mesures et des indicateurs afin d'évaluer les causes, la magnitude et l'évolution de cette crise (IPCC 2018 ; IPBES 2019; Díaz et al. 2020 ; Rounsevel et al. 2020). C'est dans ce contexte que des instances comme le GIEC Groupe d'experts intergouvernemental sur l'évolution du climat - et l'IPBES Plateforme intergouvernementale scientifique et politique sur la biodiversité et les services écosystémiques - au niveau international ont été créées. Au niveau national, de nombreuses structures permettant l'analyse des problèmes et la mise en place des solutions adaptées ont également vu le jour ${ }^{1}$. Ce contexte fait émerger de nouvelles

\footnotetext{
${ }^{1}$ En France, la loi n ${ }^{\circ} 2016-1087$ dite «pour la reconquête de la biodiversité, de la nature et des paysages» ainsi que la création de l'Office français pour la biodiversité sont des
} 
thématiques scientifiques. Ainsi, évaluer l'état global et les tendances d'évolution de la diversité biologique et culturelle et de l'environnement requiert-il de disposer d'une grande quantité de données et de les organiser en séries temporelles ou géographiques. Les collections d'histoire naturelle constituent une ressource extraordinaire dans ce contexte. Outre leur fonction originelle de référentiels pour la systématique, elles deviennent aujourd'hui un colossal gisement d'informations contribuant à l'exploration de cette problématique. Les bénéfices économiques de leur contribution, même s'ils sont encore peu évalués, pourraient être de l'ordre de plusieurs milliards de dollars (Suarez et Tsutsui 2004).

\subsection{Les collections dans la science du début du $\mathrm{XXI}^{\mathrm{e}}$ siècle}

La recherche sur les collections acquiert une place scientifique inédite du fait de son potentiel pour répondre aux grands questionnements actuels. Les spécimens de collection contiennent des informations qui permettent de reconstruire le puzzle de l'histoire de la vie, de l'homme et de l'univers et donnent des pistes pour comprendre les transformations environnementales. Les thématiques des recherches sur les collections s'en trouvent donc élargies. Les collections demeurent indispensables pour la systématique. A son tour et plus que jamais, la systématique contribue à enrichir les collections grâce au développement sans précédent des méthodes d'identification et aux inférences phylogénétiques et évolutives. Il en résulte un accroissement des identifications et des mises à disposition des nouveaux jeux de données qui facilitent l'étude du vivant dans son ensemble. La systématique crée ainsi de nouvelles formes d'association entre spécimens et informations toujours plus nombreuses. Son rôle est essentiel et indispensable en regard de toute utilisation ultérieure des spécimens.

Les collections sont de plus en plus utilisées dans deux domaines nouveaux : la recherche de nouvelles informations sur les spécimens et les analyses globales sur de grands ensembles de spécimens. Le principe de science ouverte, sur lequel la systématique a été bâtie et qui a toujours été à la base des échanges de données de collections, vient ainsi façonner nouvelles formes de mise à disposition des données. Une large gamme d'outils a été développée pour accéder à l'information résidente dans les collections. Par exemple, de nouvelles techniques sont disponibles pour identifier les parasites et commensaux (virus, bactéries, protozoaires, etc.) ou pour mesurer les contenus chimiques (taux de carbone, métaux lourds, isotopes stables, etc.) des spécimens de collection. De nouvelles approches informatiques permettent

conséquences directes de la ratification du Protocole de Nagoya, le $3^{\mathrm{e}}$ objectif principal de la Convention de la diversité biologique, qui évoque le partage juste et équitable des avantages découlant de l'exploitation des ressources génétiques. 
la mise à disposition de l'ensemble des données des collections à partir de portails uniques dits « agrégateurs».

\subsection{Nouvelles explorations grâce à la magnitude et la diversité des données des collections}

La magnitude de la diversité taxonomique, spatiale et temporelle des données hébergées dans les milliers de collections naturalistes est colossale. Les collections hébergent non seulement les types porte-noms qui permettent d'utiliser les noms scientifiques, mais aussi, très souvent, de nombreux spécimens présumés appartenir à la même espèce. La richesse de ce contenu permet donc d'étudier les questions de diversité génétique, de limites d'espèces, mais aussi de comprendre des phénomènes globaux pour lesquels un grand nombre d'échantillons est indispensable. Les nombreux exemplaires d'une même espèce constituent des réplicats génétiques, spatiaux et temporels qui permettent de tester bien des hypothèses.

La magnitude des collections se reflète également dans leur couverture spatiale gigantesque. La plupart des environnements de la planète ont été échantillonnés et comportent des spécimens correspondants dans les collections. Pour de très nombreuses localités, il existe ainsi une grande richesse de données. Des millions de spécimens retracent les milliers de missions institutionnelles ou individuelles qui ont permis d'accéder aux environnements les plus divers et les plus difficiles (figure 1.1). Ils racontent aussi l'histoire des explorations et celle des nombreuses questions scientifiques posées au cours des dernières décennies. De fait, les collections permettent d'avoir un regard d'ensemble sur la distribution du Vivant sur la planète. Aucune expédition globale ne pourrait permettre de récolter à nouveau ces millions d'informations, d'autant que la plupart des lieux ont été transformés et les environnements naturels d'autrefois ont donné naissance à des villes ou à des zones cultivées.

Un autre aspect important des collections vient du fait qu'elles abritent des spécimens récoltés à divers moments du passé, jusqu'en des temps très reculés. Les exemples les plus extraordinaires mentionnés dans ce volume sont les restes de guirlandes de fleurs trouvées sur la momie royale de Ramsès II (chapitre 12 par Quilès et al.), ou encore la collection de momies, datées entre le $\mathrm{III}^{\mathrm{e}}$ millénaire avant J.-C. et le XIX siècle de notre ère (chapitre 4 par Thomas et al.). Au sein des institutions européennes, il n'est pas rare d'avoir des collections diverses sur la plupart des biomes des cinq continents datant du XVII ${ }^{\mathrm{e}}$ siècle. Certains objets très anciens apportent des informations sur le mode de vie des peuples, leurs caractéristiques physiques, leur environnement ou leur culture. 


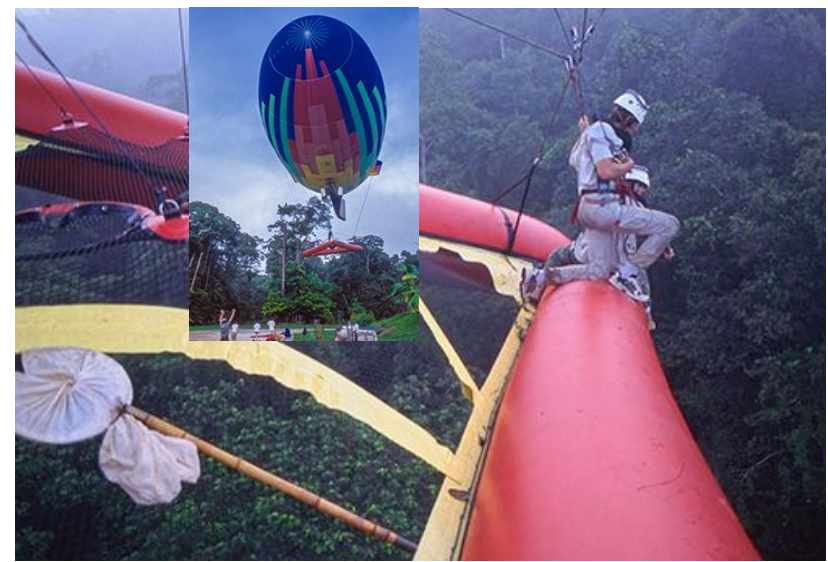

a)
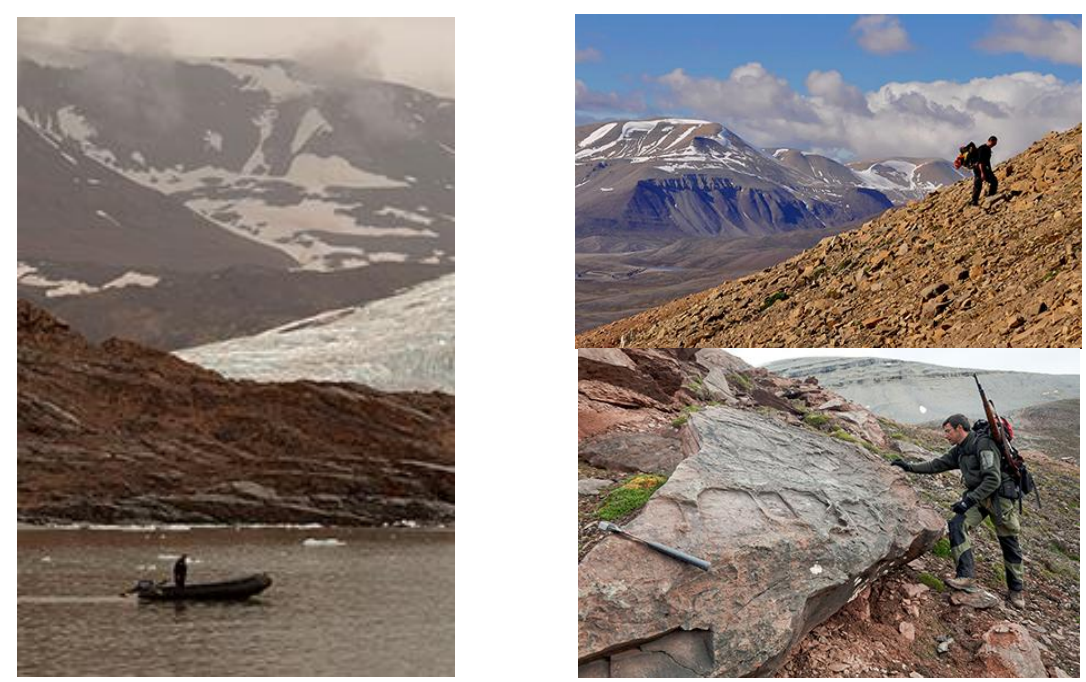

b)

Figure 1.1. a) La "luge des cimes " en Guyane, un outil pour collecter et étudier les canopées tropicales (au premier plan un filet-fauchoir d'un entomologiste pour collecter les insectes; en incrustation, une vue d'ensemble de l'engin). b) Mission au Spitzberg, écologie marine et étude des affleurements rocheux Carbonifères et Dévonien. (c) R. Garrouste, MNHN.

Mais l'intérêt principal des grandes séries temporelles est surtout de permettre de constituer ce que l'on appelle vulgairement des «points zéro », c'est-à-dire des points de référence sur l'état des populations ou des environnements avant certaines actions humaines, par exemple depuis le début de l'ère industrielle. La distribution 
des organismes peut ainsi être connue avant la destruction des habitats naturels, ou encore certains effets du changement climatique sur la biodiversité peuvent être mesurés (voir chapitre 15 par Robuchon et al. et chapitre 16 par Muller et al.). Ces connaissances sont indispensables pour affiner les propositions pour la conservation des organismes, voire même leur réintroduction comme c'est le cas à partir de la germination des graines des plantes disparues tirées des herbiers (voir chapitre 10 par Muller et al.).

\subsection{Une recherche sur collections utilisatrice et motrice}

Un tel nombre de résultats positifs pourrait laisser imaginer que les données de collections parlent d'elles-mêmes. Mais rien n'est plus inexact. Extraire l'information, faire parler ces données est une démarche scientifique qui demande de l'expertise, de l'inventivité, des connaissances sur les outils et les méthodes et des connaissances d'arrière-plan sur les questions traitées. Pour cette raison, très souvent, ces recherches sont inter- ou transdisciplinaires et réalisées dans le cadre des collaborations interinstitutionnelles, où chaque partie apporte une expertise complémentaire. Ces démarches bénéficient des stratégies collaboratives internationales, comme la création d'agrégateurs qui rassemblent les bases et données utilisées (par exemple GBIF), la création de référentiels taxonomiques qui mettent à disposition nomenclature et taxonomie, l'élaboration de systèmes collaboratifs d'analyse (R Core Team) (voir chapitre 18 par Monnet et al.), la création de bibliothèques de référence pour des études portant sur les ADN anciens (voir chapitre 13 par Gagnevin et al.), etc. Le développement ou l'adaptation des méthodes, inhérents à la démarche scientifique, font partie du quotidien de cette recherche.

Cet ouvrage a comme objectif principal de dévoiler cet univers scientifique basé sur les collections naturalistes en ce début du XXI ${ }^{\mathrm{e}}$ siècle. Un seul volume ne suffit pas pour traiter de l'ensemble de ces questions scientifiques et sociétales, mais permet d'en présenter un échantillon représentatif. Nous verrons que les collections constituent un important support pour la connaissance. Leur qualité fondamentale réside dans la conservation et dans la permanence des spécimens qui peuvent être revisités à la lumière de nouvelles questions et de nouvelles technologies. Dans cette conception de la science, les données obtenues restent toujours disponibles, structurées et ré-analysables.

Les chapitres traitent de sujets divers et se focalisent sur une problématique scientifique, un spécimen, un groupe d'organismes, un ensemble de collections. Leur point commun à tous est la reconnaissance de cette valeur des collections, révélée par la science. D'un côté, les collections servent de support pour la 
connaissance de la vie, de l'Homme et de l'Univers. De l'autre, la constitution des collections, leur raison d'être, leur gestion et leur avenir sont aussi objets des questionnements scientifiques et éthiques en perpétuel renouvellement.

\subsubsection{Pouvoir retourner à l'objet : l'une des contributions majeures des collections naturalistes}

Dans le chapitre 2, Grandcolas résume et analyse les concepts qui ont amené à la création des collections et des systèmes de classification du vivant et des minéraux. Dans cette conception, les collections sont représentatives d'une manière de faire la science basée sur la possibilité illimitée de retourner à un objet d'étude. Son analyse met en évidence un paradoxe dans la situation des collections par rapport à la science actuelle. D’une part, elles n'ont jamais été autant nécessaires pour éclairer des problématiques scientifiques dans l'urgence. D'autre part, elles risquent d'être rapidement remplacées par une science basée sur des milliards de données produites par des méthodes qui ne permettent aucun retour au spécimen, comme les données des sciences participatives ou de la métagénomique. Le risque est de produire ainsi une connaissance sans aucune traçabilité ou possibilité de révision ou de réfutation, tout le contraire de ce qui a été construit pendant plusieurs siècles de recherches en histoire naturelle. Il y a donc urgence à trouver des stratégies de réconciliation entre ces différents types de données, que certains considèrent respectivement comme «quality data » versus « Big Data».

Pouvoir réexaminer les objets et les documents associés constitue donc un aspect indissociable des recherches sur les collections et ce sujet fait donc partie de la plupart des études de cet ouvrage (pour un aperçu à plus large échelle, voir le chapitre 15 par Robuchon et al.). Néanmoins, les trois chapitres suivants illustrent bien ce caractère des recherches sur les collections. La redécouverte du diamant bleu, l'une des deux gemmes extraordinaires de Louis XIV, représente sans doute un bel exemple à cet égard. Volé en 1792, ce diamant était considéré comme perdu jusqu'à très récemment. Une analyse détaillée d'une riche documentation, la redécouverte d'un moulage, et la possibilité d'examiner le diamant modifié après le vol ont permis de confirmer d'anciennes hypothèses sur sa localisation, mais aussi de reconstituer une copie en zircon avec plusieurs de ses caractéristiques uniques (voir chapitre 3 par Farges).

Le deuxième exemple est l'étude de la momie Chachapoya (voir chapitre 4 par Thomas et al.). Cet objet, qui fait partie de l'histoire muséale et scénique du Musée de l'Homme et qui a inspiré l'un des tableaux les plus remarquables de l'histoire de l'art de la fin du XIX ${ }^{\mathrm{e}}$ et du début $\mathrm{XX}^{\mathrm{e}}$ siècle - Le Cri d'Edvard Munch - participe maintenant de l'histoire de la science moderne sur les peuples amérindiens. Les 
études récentes et l'emploi des nouvelles technologies ont permis d'examiner l'intérieur du corps et de comprendre comment cet individu a vécu ainsi que les causes de son décès.

Le troisième exemple concerne des nouvelles études sur les crânes humains, probablement l'un des objets de collection les plus examinés de l'histoire de la science (voir le chapitre 5 par Friess et Galand). Cette étude montre comment les nouvelles méthodes de morphométrie 3D ont permis d'apporter un regard nouveau et de contribuer à mieux comprendre des problématiques importantes sur l'évolution de la diversité humaine : le rapport entre la forme du crâne et la distribution géographique et la diversité linguistique.

\subsubsection{Les collections au cœur des recherches très innovantes grâce aux nouvelles technologies}

Un autre aspect des utilisations innovantes des collections est l'importance des technologies pour accéder à des informations qui n'ont pas pu être exploitées auparavant. Le chapitre 6 (Invernón et al.) montre comment une étude des espèces hyperaccumulatrices des métaux et des terres rares dans les planches d'herbier a été possible grâce à la spectrométrie de fluorescence des rayons X (XRF). Les résultats obtenus indiquent comment l'herbier permet d'identifier non seulement des espèces utiles pour la restauration des sites contaminés par des métaux lourds, mais aussi de rechercher des lieux où la concentration de certains métaux et terres rares peut être importante. Ces technologies de fluorescence ainsi que la microtomographie à rayons $\mathrm{X}$ ont également un grand intérêt pour l'étude des fossiles, car ils permettent de visualiser des structures invisibles à œil nu ou par d'autres méthodes traditionnelles (voir chapitre 7 par Charbonnier et Forel). Des recherches extrêmement diverses sont possibles grâce à ces nouvelles technologies (systématique, étude du développement ou paléogéographie ancienne, etc.).

Une autre facette des nouveaux questionnements et des nouvelles possibilités technologiques a trait à la création de collections innovantes. Des collections qui, dans un premier temps, sont destinées à répondre à des demandes bien spécifiques, ouvrent un nouvel univers de connaissances et d'expérimentations. Un excellent exemple est illustré dans le chapitre 8 (Duperron et al.) avec la collection de souches vivantes de cyanobactéries et de microalgues, constituée pour servir de référence aux tests écotoxicologiques et aux diagnostics environnementaux. Duperron et al. montrent que cette collection, outre son utilisation en systématique, présente un intérêt majeur dans la recherche facilitée par les approches «-omics » à haut débit. Par exemple, elle permet de mieux étudier les interactions entre organismes, car le maintien des spécimens en collection maintient également les cyanosphère et 
phycosphère associées. Elle permet aussi la recherche des métabolites bioactifs, avec, principalement, une finalité pharmaceutique, qui sont explorés de manière de plus en plus fine grâce aux nouvelles technologies. Le chapitre 9 (Gerbault-Seureau et Dutrillaux) représente un autre exemple à travers les collections de cellules et tissus cryoconservés de vertébrés homéothermes. Cette collection très riche, mais encore peu exploitée, représente une source importante de matériel cellulaire, avec son ADN récent et son ARN des fibroblastes, disponibles pour la recherche. Conserver les tissus et cellules d'espèces protégées est d'autant plus intéressant que ces espèces vulnérables ont de petits effectifs. Des études sur des tissus vivants de ces espèces peuvent être conduites à répétition, après culture cellulaire, sans aucune nuisance pour les individus vivants.

Si les cellules et les tissus cryoconservés ne permettent pas de faire revivre les animaux homéothermes, les graines gardées dans des collections naturalistes offrent cette possibilité. Des scientifiques se sont ainsi fixés pour but de faire revivre des espèces disparues dont des graines sont conservées dans l'herbier (voir chapitre 10 par Muller et al.). Cette démarche innovante requiert évidemment une expertise sur la physiologie de la reproduction des espèces ciblées. Ce chapitre 10 expose donc les premiers résultats d'un projet ambitieux et précurseur d'un type de recherche probablement amené à devenir très fréquent pendant les prochaines décennies.

\subsubsection{Une ressource pour la recherche sur les changements globaux}

Un des principaux défis dans l'étude des changements globaux est l'établissement de points de référence à partir desquels nous pouvons constater la magnitude et la direction des changements en cours. Les changements globaux, et notamment les changements climatiques, se mesurent à des échelles de temps beaucoup plus longues que celles de nos systèmes d'observation contemporains. Par exemple, la plupart des données climatiques dont nous disposons proviennent de stations mises en place à partir de la seconde moitié $\mathrm{du} \mathrm{Xx}^{\mathrm{e}}$ siècle. Ainsi les études sur des collections, qu'elles soient paléontologiques, archéologiques ou actuelles, cherchent donc à définir et calibrer des proxys pour comprendre ces changements.

La recherche sur les changements climatiques nécessite de replacer ces proxys dans le contexte de l'évolution des climats depuis la dernière vingtaine ou centaine de milliers d'années. Dans le chapitre 11, Moreno et Bartolini montrent l'une de ces approches : la recherche des proxys climatiques et de salinité dans les sédiments marins (de quelques dizaines de milliers d'années au dernier million d'années). Cette recherche est basée sur des collections nouvelles de carottes océaniques, constituées principalement à partir de 1970. Ce chapitre dévoile les défis inhérents à la constitution et à la conservation, ainsi qu'à l'analyse et à la compréhension des 
données provenant d'une ressource aussi extraordinaire. Dans le même esprit, Quilles et al. (chapitre 12) montrent comment les collections peuvent donner accès aux teneurs en gaz atmosphériques avant un changement important du paysage. Il s'agit de calibrer la courbe de radiocarbone avant la construction du barrage d'Assouan en Égypte, qui a fortement impacté cette région, en tenant compte des effets des inondations périodiques du Nil. Les analyses indiquent que les planches d'herbier des $\mathrm{XVII}^{\mathrm{e}}$ et $\mathrm{XVIII}$ e siècles permettent de détecter les teneurs de ${ }^{14} \mathrm{C}$ dans l'atmosphère de cette période. Cette étude contribue donc à affiner des datations archéologiques sur une période historique de plusieurs millénaires, enjeu majeur pour la connaissance de l'histoire de l'Égypte ancienne. Ces deux projets révèlent combien les collections peuvent être au cœur de recherches fortement transdisciplinaires.

Les deux chapitres suivants traitent des recherches sur la dispersion des pathogènes de cultures et des espèces exotiques envahissantes, problèmes associés à la globalisation de l'agriculture et au transport des produits agricoles divers à très longue distance. L'étude de Gagnevin et al. (chapitre 13) explore l'utilisation des herbiers pour l'étude de Xanthomonas citri pv. citri qui infecte les agrumes et les géminivirus qui attaquent le manioc et la patate douce. Ces pathogènes ont un fort impact sur la production agricole dans les régions de l'océan Indien ou de l'Afrique subsaharienne. Leurs résultats montrent comment de nouvelles méthodes moléculaires et génomiques permettent d'accéder aux informations résidentes dans les herbiers, riches en espèces d'intérêt agricole. Ces analyses permettent de tracer l'origine, l'histoire évolutive et les routes de dispersion des maladies qui peuvent avoir un impact majeur sur l'économie régionale. Le rôle des collections pour comprendre les invasions biologiques est illustré par Villemant et al. (chapitre 14), à propos du frelon asiatique Vespa velutina, la première espèce exotique de Vespidae acclimatée en Europe. Leur étude souligne l'importance des données des collections pour connaître l'origine des lignées invasives, la structure de la diversité dans son aire d'origine, et ainsi permettre de faire des prédictions plus robustes sur les aires potentielles d'invasion.

Ainsi qu'il a déjà été mentionné, utiliser les collections pour répondre à des questions ciblées requiert des ajustements méthodologiques. Parfois, les données des collections ne suffisent pas pour mener une étude à bien. Néanmoins, leur prise en compte permet a minima de recadrer le sujet et d'ajuster le protocole. Cet aspect itératif tellement fondamental de la recherche scientifique est bien souligné par l'étude de Robuchon et al. (chapitre 15). Les données existantes ne permettent pas d'aboutir à des conclusions claires. Cependant, une fois complétées par des collectes ciblées sur les mêmes espèces aux mêmes endroits, elles peuvent représenter un 
solide point de référence passé pour les recherches sur les changements de communautés et les extinctions locales.

Malgré leur nombre, les chapitres présentés dans cet ouvrage ne nous offrent qu'un aperçu de la diversité des recherches sur les changements globaux pour lesquelles les collections représentent une source incontournable de données. La revue de Muller et al. (chapitre 16) portant sur les herbiers nous confirme qu'il existe déjà une diversité d'approches surprenante, même pour ce seul type de collections. La contribution des collections à la compréhension des effets des changements globaux n'en est manifestement qu'à ses débuts.

\subsubsection{Concevoir la science du futur sur les collections}

Comme tous ces exemples le montrent, la recherche basée sur les collections est à la fois utilisatrice pour répondre à des questions spécifiques et incitatrice en termes de nouvelles conceptions pour les collections. Les chapitres de cet ouvrage mettent en évidence différents savoir-faire qui enrichissent aussi les collections.

Dans le chapitre 17, Garrouste propose une réflexion sur le travail du naturaliste, avec l'association d'images numériques 2D, 3D et même 4D (films, mouvements) aux spécimens récoltés. Ces outils permettent de conserver facilement des informations sur des spécimens vivants (couleurs précises, mouvements, comportements, interactions). L'association de ces informations aux spécimens de collections permet de faire un saut conceptuel, tant elles sont indispensables à l'écologie, la physique des mouvements, la bio-inspiration ou les études de l'évolution. Malgré ces avantages, l'auteur mentionne deux contextes où ces utilisations sont, respectivement, exacerbées et négligées, et réduisent ainsi leur potentiel scientifique : l'utilisation des photos comme type porte-nom lors de la description des espèces et les limitations dans l'utilisation des images pour les identifications dans les projets de science participative.

De nos jours, une partie importante de la recherche sur les collections naturalistes consiste à valider les données ou à neutraliser leurs biais en regard des questions posées. Dans le chapitre 18, Monnet et al. discutent de la mise à disposition et de l'utilisation des données via des portails ou agrégateurs de données, dans le cadre d'un système de traçabilité depuis le spécimen de collection jusqu'au dernier d'une série d'utilisateurs. En effet, au cours des deux dernières décennies, les institutions ont consenti d'énormes efforts afin de rendre disponibles leurs données et le nombre de recherches qui les utilisent augmente exponentiellement. Les publications scientifiques ont aussi un rôle fondamental, en recommandant ou en exigeant que les données utilisées soient disponibles. Malgré tout, l'ensemble des 
pratiques a besoin d'être revu avec rigueur, en constituant des pipelines de traitement et d'utilisation de données qui soient plus en accord avec des principes de validation et de traçabilité. Le suivi de l'évolution des connaissances sur les spécimens, l'association entre les diverses informations obtenues pour le même spécimen (Gene Bank Accession Number, GBIF ID, etc.), ainsi que celui de la reprise des données ré-échantillonnées par d'autres groupes de recherche restent encore très limités. Si l'on considère que les portails et agrégateurs de bases de données sont conçus pour durer dans le temps et qu'ils ne peuvent pas être reconstruits à chaque nouvelle étude, il est clair que de nouvelles procédures doivent être mises en œuvre à cet égard.

Questionner les collections, leurs valeurs, leurs besoins d'entretien et de continuité font partie du quotidien des scientifiques chargés des collections. Ce lien amène aussi à de nombreux questionnements non pas seulement sur comment ajouter des informations et en assurer la traçabilité, comme le proposent les études des chapitres 17 (Garrouste) et 18 (Monnet et al.), mais également sur leur raison d'être et leur devenir. L'étude de Bahuchet (chapitre 19) explore cette problématique à travers une analyse des collections bioculturelles. La question de fond « faut-il des collections bioculturelles?» est posée en regard des objets indispensables pour témoigner des relations des peuples avec la nature. Cette problématique concerne en fait tous les types de collections. Elle nous montre que les décisions relatives à une collection ne peuvent être prises sans considérer le contexte scientifique ayant donné lieu à leur constitution.

Cet ouvrage se termine avec une étude sur la conservation, une thématique de recherche indissociable de l'existence des collections. Rouchon (chapitre 20) détaille des pratiques de conservation et leurs changements au fil du temps, particulièrement dans des cas pour lesquels l'objet à conserver pose des défis méthodologiques et techniques. Ces exemples dévoilent l'ampleur du problème de la conservation. Comment éviter les altérations des objets, qu'elles soient d'ordre physique, ou résultantes de réactions chimiques ou d'une activité biologique ? À travers la question «pourquoi conserver? », l'auteure souligne les différentes valeurs que nous accordons aux collections ou à certains objets, et leurs transformations avec les changements d'utilisations et de connaissances acquises sur les objets. Mieux conserver implique ainsi de mieux les connaître.

La conclusion de cet ouvrage (chapitre 21) s'interroge sur les contributions des collections pour les sciences du futur. Rétrospectivement, il aurait été difficile à la fin du XIX ${ }^{\mathrm{e}}$ siècle d'imaginer la plupart des recherches actuellement menées sur les collections. Il en va de même de notre capacité à prévoir en détails les besoins sociétaux et les regards scientifiques pour le prochain siècle ou le suivant. 
Néanmoins, deux éléments peuvent nous y aider : d'une part, considérer l'ensemble des pratiques et des principes qui ont permis la conservation des informations riches résidentes dans les collections; d'autre part, apprécier l'évolution de la systématique, la science qui a servi de fil rouge pour l'établissement d'une grande partie des collections. Ces deux éléments nous montrent déjà tout le potentiel futur de la recherche sur collections.

\subsection{Bibliographie}

Amano, T., Sandel, B., Eager, H., Bulteau, E., Svenning, J.-S., Dalsgaard, B., Rahbek, C., Davies, R.G., Sutherland, W.J. (2014). Global distribution and drivers of language extinction risk [En ligne]. Proceedings of the Royal Society B: Biological Sciences, 281(1793), 17-19. Disponible à l'adresse : https://doi.org/10.1098/rspb.2014.1574.

Barnosky, A.D., Matzke, N., Tomiya, S., Wogan, G.O.U., Swartz, B., Quental, T.B., Marshall, C., McGuire, J.L., Lindsey, E.L., Maguire, K.C., Mersey, B., Ferrer, E.A. (2011). Has the Earth's sixth mass extinction already arrived? [En ligne]. Nature, 471(7336), 51-57. Disponible à l'adresse : https://doi.org/10.1038/nature09678.

Barnosky, A.D., Hadly, E.A., Bascompte, J., Berlow, E.L., Brown, J.H., Fortelius, M., Getz, W.M., Harte, J., Hastings, A., Marquet, P.A., Martinez, N.D., Mooers, A., Roopnarine, P., Vermeij, G., Williams, J.W., Gillespie, R., Kitzes, J., Marshall, C., Matzke, N., Mindell, D.P., Revilla, E., Smith, A.B. (2012). Approaching a state shift in Earth's biosphere [En ligne]. Nature, 486(7401), 52-58. Disponible à l'adresse : https://doi.org/10.1038/nature11018.

Carlson, C.J. (2020). From PREDICT to prevention, one pandemic later [En ligne]. The Lancet. Disponible à l'adresse : https://doi.org/10.1016/ S2666-5247(20)30002-1.

Ceballos G., Ehrlich, P.R., Barnosky, A.D., García, A., Pringle, R.M., Palmer, T.M. (2015). Accelerated modern human-induced species losses: Entering the sixth mass extinction [En ligne]. Science Advances, 1(5). Disponible à l'adresse : https://doi.org/10.1126/sciadv.1400253.

Díaz, S. et al. (2020). Set ambitious goals for biodiversity and sustainability [En ligne]. Science, 370(6515), 411-413. Disponible à l'adresse: https://doi.org/10.1126/science. abe1530.

Gorenflo, L.J., Romaine, S., Mittermeier, R.A., Walker-Painemilla, K. (2012). Co-occurrence of linguistic and biological diversity in biodiversity hotspots and high biodiversity wilderness areas [En ligne]. Proceedings of the National Academy of Sciences of the United States of America, 109(21), 8032-8037. Disponible à l'adresse : https://doi.org/10.1073/pnas.1117511109.

Hoekstra, A.Y., Wiedmann, T.O. (2014). Humanity's unsustainable environmental footprint. Science, 344, 1114-1117. 
Hoekstra, A.Y., Mekonnen, M.M., Chapagain, A.K., Mathews, R.E., Richter, B.D. (2012). Global monthly water scarcity: Blue water footprints versus blue water availability [En ligne]. PLoS One, 7(2). Disponible à l'adresse: https://doi.org/10.1371/ journal.pone. 0032688 .

IPBES (2019). Summary for policymakers of the global assessment report on biodiversity and ecosystem services [En ligne]. Rapport, Intergovernmental Science-Policy Platform on Biodiversity and Ecosystem Services, Bonn. Disponible à l'adresse : https://doi.org/10.5281/zenodo.3553579.

IPCC (2018). Summary for Policymakers. Dans Global Warming of $1.5^{\circ} \mathrm{C}$. An IPCC Special Report on the impacts of global warming of $1.5^{\circ} \mathrm{C}$ above pre-industrial levels and related global greenhouse gas emission pathways, in the context of strengthening the global response to the threat of climate change, sustainable development, and efforts to eradicate poverty, Masson-Delmotte, V., Zhai, P., Pörtner, H.-O., Roberts, D., Skea, J., Shukla, P.R., Pirani, A., Moufouma-Okia, W., Péan, C., Pidcock, R., Connors, S., Matthews, J.B.R, Chen, Y., Zhou, X., Gomis, M.I., Lonnoy, E., Maycock, T., Tignor, M., Waterfield, T. (dir.). World Meteorological Organization, Genève.

Rounsevell, M.D.A., Harfoot, M., Harrison, P.A., Newbold, T., Gregory, R.D., Mace, G.M. (2020). A biodiversity target based on species extinctions. [En ligne]. Science, 368(6496), 1193-1195. Disponible à l'adresse : https://doi.org/10.1126/science.aba6592

Steffen, W., Richardson, K., Rockström, J., Cornell, S.E., Fetzer, I., Bennett, E.M., Biggs, R., Carpenter, S.R., de Vries, W., de Wit, C.A., Folke, C., Gerten, D., Heinke, J., Mace, G.M., Persson, L.M., Ramanathan, V., Reyers, B., Sörlin, S. (2015). Planetary boundaries: Guiding human development on a changing planet [En ligne]. Science, 347(6223). Disponible à l'adresse : https://doi.org/10.1126/science.1259855.

Suarez, A.V., Tsutsui, N.D. (2004). The Value of Museum Collections for Research and Society [En ligne]. BioScience, 54(1), 66-74. Disponible à l'adresse : https://doi.org/10.1641/0006-3568(2004)054[0066:TVOMCF]2.0.CO;2. 